\title{
Gaming enhances learning-induced plastic changes in the brain
}

\section{Authors}

Katja Junttila *,a , Anna-Riikka Smolander a , Reima Karhila ${ }^{\mathrm{b}}$, Anastasia Giannakopoulou ${ }^{\mathrm{c}}$, Maria Uther ${ }^{\mathrm{d}}$, Mikko Kurimo ${ }^{\mathrm{b}}$, Sari Ylinen ${ }^{\mathrm{a}, \mathrm{e}}$

${ }^{a}$ Cognitive Brain Research Unit, Department of Psychology and Logopedics, Faculty of Medicine, University of Helsinki, Finland

${ }^{\mathrm{b}}$ Department of Signal Processing and Acoustics, Aalto University, Finland

${ }^{\mathrm{c}}$ School of Psychology, University of Bedfordshire, United Kingdom

${ }^{\mathrm{d}}$ Department of Psychology, University of Wolverhampton, United Kingdom

${ }^{\mathrm{e}}$ CICERO Learning, Faculty of Educational Sciences, University of Helsinki, Finland

* Corresponding author:

Katja Junttila

Cognitive Brain Research Unit

Faculty of Medicine

POB 21

FI-00014 University of Helsinki

E-mail address: katja.junttila@helsinki.fi

\begin{abstract}
Learning is increasingly assisted by technology. Digital games may be useful for learning, especially in children. However, more research is needed to understand the factors that induce gaming benefits to cognition. In this study, we investigated the effectiveness of digital game-based learning approach in children by comparing the learning of foreign speech sounds and words in a digital game or a non-game digital application with equal amount of exposure and practice. To evaluate gaming-induced plastic changes in the brain function, we used the mismatch negativity (MMN) brain response that reflects the activation of long-term
\end{abstract}


memory representations for speech sounds and words. We recorded auditory event-related potentials (ERPs) from 37 school-aged Finnish-speaking children before and after playing the "Say it again, kid!” (SIAK) language-learning game where they explored game boards, produced English words aloud, and got stars as feedback from an automatic speech recognizer to proceed in the game. The learning of foreign speech sounds and words was compared in two conditions embedded in the game: a game condition and a non-game condition with the same speech production task but lacking visual game elements and feedback. The MMN amplitude increased between the pre-measurement and the postmeasurement for the word trained with the game but not for the word trained with the nongame condition, suggesting that the gaming intervention enhanced learning more than the non-game intervention. The results indicate that digital game-based learning can be beneficial for children's language learning and that gaming elements per se, not just practise time, support learning.

\section{Keywords}

\section{digital game-based learning, gaming, foreign-language learning, automatic speech recognition}

\section{Introduction}

Computer-assisted language learning (CALL) has been found to be even more effective for foreign-language learning than instruction without technology use (Grgurović et al., 2013). Digital game-based learning is a variant of CALL and it can be an effective way to learn foreign languages (e.g. Aghlara \& Tamjid, 2011; Chen et al., 2018; Franciosi, 2017; Hsu, 2017; Liu \& Chu, 2010; Sandberg et al., 2014; Tsai \& Tsai, 2018; see reviews: Acquah \& Katz, 2020; Zou et al., 2019), albeit it has also been suggested that watching someone play can be even more effective for learning because of the increased cognitive load of actually playing the game (deHaan et al., 2010). In addition to learning what is practiced in the game, playing some types of digital (video) games can also promote learning transferable skills (Bavelier \& Davidson, 2013; Eichenbaum et al., 2014). Learners often find learning by playing both enjoyable and motivating (Peterson, 2010a, 2010b; Yip \& Kwan, 2006; Young $\&$ Wang, 2014). In addition to preferring digital-game based learning over regular activitybased classroom learning, students are also found to learn vocabulary more effectively with 
digital games (Yip \& Kwan, 2006). Rigorous studies comparing the effectiveness of different features of the game or studies comparing learning with a game and learning the same content with other types of media are rarely conducted, yet they are much needed to better understand the best practices to support learning (Bavelier \& Davidson, 2013; Mayer, 2015). In particular, although positive effects of digital game-based language-learning have been shown, it is not yet understood whether these effects are due to motivation increasing the practicing time (Sandberg et al., 2011; Tejedor-García et al., 2020), or the game-like elements themselves enhancing learning.

To this end, the aim of this study was to determine the effectiveness of computer-game based language learning approach on children's foreign-language learning, while controlling for the gaming time. By presenting equal number of repetitions of words to be learned with the game training condition and a non-game training condition and thus ensuring that potentially better learning results could not be caused by more practice and a larger number of repetitions of the words to be learned, we aimed do examine whether gaming (or some of its elements) per se enhances learning.

We investigated the effects of digital game-based language learning and plastic changes in the brain as a result of gaming by measuring auditory event-related potentials (ERPs), more specifically, the mismatch negativity (MMN) component. The MMN response was first found to be elicited to auditory stimuli that deviated from the other sounds presented in a same sequence (Näätänen et al., 1978). This is typically termed an 'oddball' paradigm, where a stimulus sequence consists of a high probability standard sound that is repeated and occasionally replaced by a low probability deviant sound. Later, Näätänen and colleagues (1997) showed that the MMN is enhanced by the activation of long-term memory traces for native-language speech sounds. Pulvermüller and colleagues (2001), in turn, demonstrated that the MMN reflects long-term memory traces for individual spoken words, as is shown by an increase of its amplitude. Since the MMN is an automatic response, this indicates that the memory representations for speech sounds and words are activated automatically even without focused attention. The increased amplitude of the MMN response may also reflect learning effects (for a review, see Näätänen et al., 2007). For example, a study by Ylinen and colleagues (2010) has shown that training can induce changes in automatic processing of foreign speech sounds, and thus enhance the ability to distinguish foreign speech sounds that resemble each other. In particular, while native Finnish speakers' MMN responses to an 
English vowel contrast were significantly smaller than those of native English speakers' MMNs at pre-test, after training no such difference was found and the Finn's pre- and posttraining MMNs differed from each other significantly. Importantly, the MMN changes were accompanied by improved behavioural categorisation of the target vowels in the Finns. Together, behavioural and brain indices suggested robust learning effects.

Because MMN elicitation does not require direct attention towards the stimuli (Fitzgerald \& Todd, 2020; Näätänen et al., 1978), it is an excellent tool to investigate learning effects especially in children (for a review, see Cheour et al., 2000). Therefore, we used MMN to investigate the effectiveness of game-based learning in children. We compared two different learning conditions: a game condition and a non-game condition. We hypothesised that the children's MMN responses will show a larger increase with the game condition if gaming enhances learning (for training effects see Ylinen et al., 2010; for gaming effect see Baker et al., 2015; Hong et al., 2017; Yip \& Kwan, 2006). Also, we aimed to investigate the contribution of right and left temporal sources to the learning of foreign speech sounds and words. Although the left hemisphere is typically associated with language-related processing (Näätänen et al., 1997), the right hemisphere has been shown to contribute more to foreignlanguage word processing in children (Nora et al., 2017; S. Ylinen et al., 2019). Therefore, we expected right hemisphere sources to be stronger than the left hemisphere sources.

\section{Materials and Methods}

\subsection{Ethics}

The study was approved by the University of Helsinki Ethical Review Board in the Humanities and Social and Behavioural Sciences. Participation was voluntary. Each participants' guardian signed a written informed consent and the participants gave their oral informed consent before participating. The participants were compensated with one cinema ticket per hour of participating. The study was conducted in accordance with the Declaration of Helsinki. 


\subsection{Participants}

Participants were 7 to 11 year old $(\mathrm{M}=9.53, \mathrm{SD}=0.77)$ children $(\mathrm{N}=37,19$ girls $)$. To be included in the study, the participants had to meet the following criteria: they had to be between 7 and 11 years of age, a monolingual native Finnish speaker, no developmental disorders, no language disorders, no learning disorders, no head injuries, normal hearing, and normal vision or vision corrected to normal with eyeglasses. The participants were screened with behavioural pre-tests and had to meet the following criteria to be included in the study: a minimum of 6 standard points in each of the Wechsler Intelligent Scale for Children IV (WISC-IV; Wechsler, 2010) subtests included in the study (Block Design, Digit Span, Coding, and Vocabulary). Out of the 42 children who volunteered, 37 fulfilled the inclusion criteria and were included in the study.

All participants attended the Finnish comprehensive school and 28 of them studied English at school. According to their guardians' reports, two children did not know any English, 18 knew a few English words, 17 could understand simple sentences in English, and none of the children could understand and speak English fluently.

\subsection{Experimental design}

\subsubsection{Behavioural tests}

To ascertain whether the children knew the target words before playing the game, or whether they learned them during the gaming, each participant's knowledge of the words used in the EEG experiment was tested before the first EEG session and again after training. The words and pseudowords used as stimuli for the EEG experiment were presented one at a time through headphones. After each word the child was asked whether the word sounded familiar to them. If the child thought the word was familiar, they were also asked what the word means.

Participants' cognitive, phonological, and literacy skills were assessed with standardised psychological tests (the phonological and literacy skills will be reported elsewhere) to determine that they fulfilled the inclusion criteria. During the skill testing, the participant sat in a quiet room with the researcher. The testing took one hour. Wechsler Intelligent Scale for 
Children IV (WISC-IV; Wechsler, 2010) and in particular its subtests Block Design, forward and backward Digit Span, Coding, and Vocabulary were used to assess perceptual reasoning skills, auditory short-term memory, eye hand coordination and processing speed, and vocabulary, respectively.

\subsubsection{EEG Stimuli}

For stimulus recordings, we chose English words with dental fricative phonemes (voiceless $/ \theta /$ and voiced /ð/) that do not belong to the Finnish phonology and were thus expected to be novel and relatively difficult for native Finnish speakers. The recordings were conducted in a sound-attenuated recording studio where native English speakers pronounced a list of English words and minimal-pair pseudowords several times. The most representative exemplars were chosen for the EEG experiment. The final stimuli presented during the EEG recording were

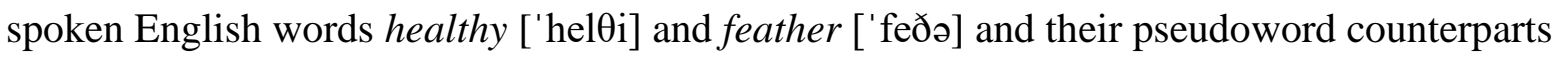
healty* ['helti]* and feder* ['fedə]* (Figure 1). The stimulus words were chosen so that most children in the target group of the study would not know their meanings. Commonly used English schoolbooks for the age group of the study were checked and words that were not included in their syllabus were chosen. The stimuli were modified using Praat software (Boersma \& Weenink, 2010). The stimuli were cross-spliced at zero-crossings, and the first $200 \mathrm{~ms}$ of the word healthy and the first $220 \mathrm{~ms}$ of the word feather were used as the beginnings of the pseudowords healty* and feder*, respectively (Figures $1 \& 3$ ). The pitch contour of healthy was adopted to healty* and the pitch contour of feather was adopted to feder*. Thus, the beginnings of healthy and healty*, as well as feather and feder* were identical, and therefore it was not possible to differentiate the pseudoword from the word before the last two phonemes of the words. 

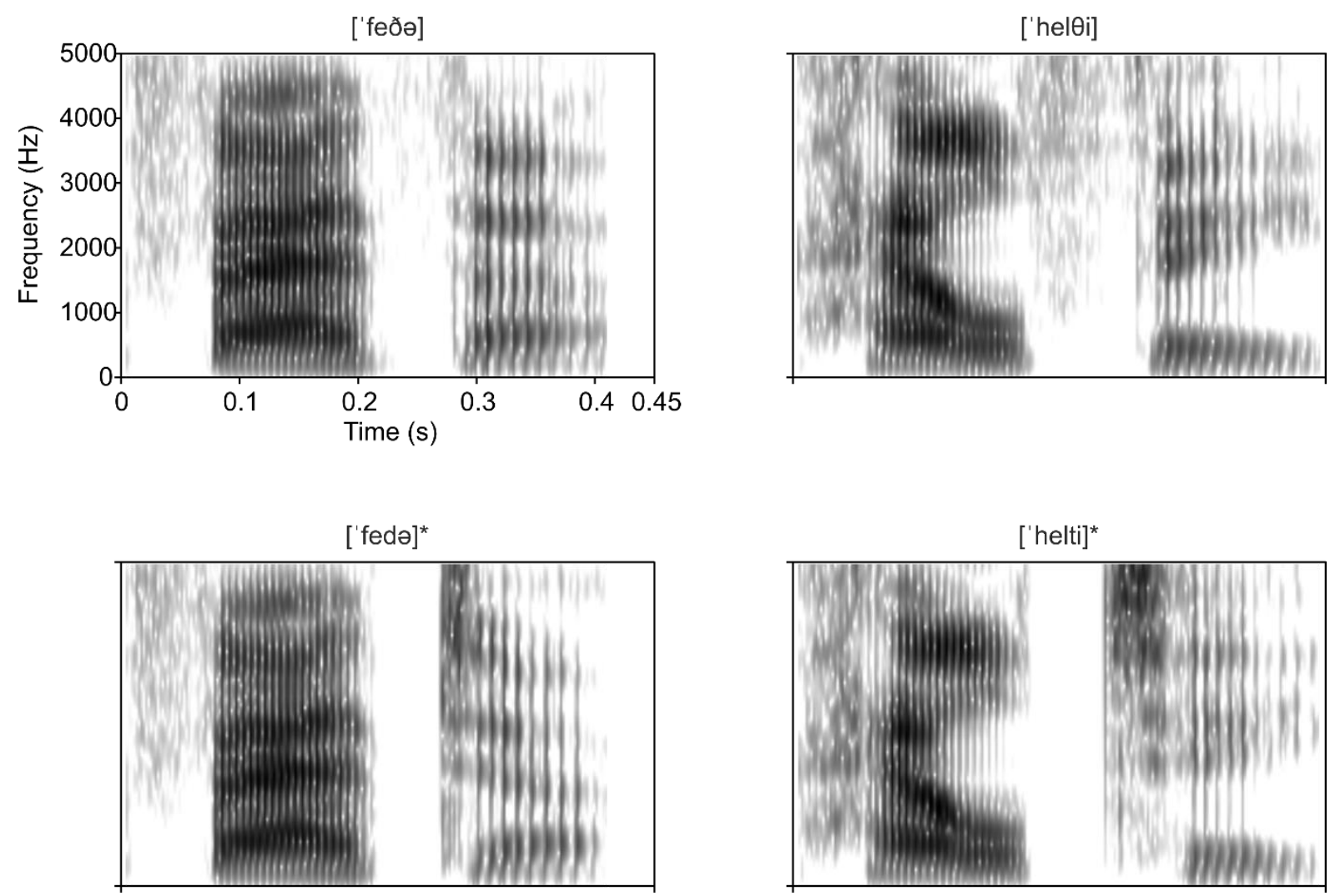

Figure 1. Spectrograms of the stimuli.

\subsubsection{EEG recordings and data processing}

All children participated in at least two EEG measurements, which were conducted before and after game-based training. Between these measurements, the participants played a language learning game. In addition, to control for the effects of repeated EEG measurements and out-of-game exposure to English, 22 of the children participated in an additional measurement that took place on average five weeks (an equal amount of time as between the two last measurements) prior to the before-training measurement (this extra measurement is referred as a baseline measurement).

EEG was recorded from 64 channels and additional electrodes on the mastoids using BioSemi ActiveTwo system (BioSemi Inc., Amsterdam, The Netherlands) with Biosemi ActiView Version 7.07 EEG acquisition software. Horizontal eye movements were monitored with two electrodes attached near the outer canthi of the eyes. Vertical eye movements were monitored with an electrode placed under the left eye. One additional electrode was attached on the tip of the nose for online reference. During the measurement, 
the participants sat on a comfortable chair in a sound and electrically attenuated room and watched a muted film of their choice with subtitles on. They were instructed to concentrate on the film and to ignore the auditory stimuli. The stimulus sequences were presented binaurally via headphones at a comfortable hearing level of $60 \mathrm{~dB}$.

The EEG experiment included four different stimulus sequences: two oddball stimulus sequences containing deviant and standard stimuli and two sequences containing only standard stimuli. In one oddball sequence, a standard pseudoword feder $*(p=0.8)$ was occasionally replaced by a deviant word feather $(\mathrm{p}=0.2)$. In the other oddball sequence, the standard pseudoword was healty* $(\mathrm{p}=0.8)$ and the deviant word was healthy $(\mathrm{p}=0.2)$. Each deviant stimulus was presented 120 times and each standard stimulus 480 times in the deviant sequences. In the two separate standard sequences, the words feather and healthy were presented 180 times each ( $\mathrm{p}=1$ for each). Inter-stimulus interval (ISI; offset to onset) was 500 $\mathrm{ms}$ in all sequences. The order of the sequences was randomised for each participant and for each measurement session. All stimulus sequences were presented by using Presentation ${ }^{\circledR}$ software (Version 17.2, Neurobehavioral Systems, Inc., Berkeley, CA, www.neurobs.com). The EEG measurement took two hours, including preparation.

After the recording, the EEG data were processed with BESA Research 7.0 software (BESA $\mathrm{GmbH}$, Gräfelfing, Germany). The data were re-referenced to the average of mastoids to improve the signal-to-noise ratio, and bad channels were interpolated. Then the data were filtered with a band pass of $1.5-20 \mathrm{~Hz}$ (slope $24 \mathrm{~dB}$ /octave; a strict high-pass was used to reduce slow drifts in children's EEG signal beyond the MMN frequency range, see Kalyakin et al., 2007; Nenonen et al., 2005; Picton et al., 2000; Sinkkonen \& Tervaniemi, 2000). Eye movements were corrected using BESA Research Artifact Correction that is based on the spatial filtering method. Data were epoched using a -100 to $800 \mathrm{~ms}$ time window (from stimulus onset), epochs with artifacts exceeding $\pm 75 \mu \mathrm{V}$ as well as 5 first epochs of each sequence were rejected, and the remaining epochs were averaged for each stimulus type separately. To examine the effects of game-based learning, we formed grand-average waveforms from ERPs for the stimulus words with phonemes trained with game condition and from the ERPs for the stimulus words with phonemes trained with the non-game condition. Note that to eliminate possible differences in difficulty between the two target phonemes, the participants were divided into two groups and words with the two target phonemes were assigned to game and non-game conditions in a counterbalanced manner in 
these groups. Consequently, the ERP waveforms were constructed by combining responses to both kinds of target words for each condition. Since we averaged the responses to the two items with slightly different divergence points $(20 \mathrm{~ms})$, the resulting MMN responses may be slightly wider and flatter than the responses to stimuli with identical timing would have been. This does not, however, affect the comparison of game and non-game conditions, because the effect is the same for both conditions.

The MMN responses were quantified from deviant-minus-standard waveforms that were created by subtracting responses to the same words presented as standards in standard-only sequences from the deviant stimuli of the oddball sequences. Fronto-central electrode sites F3, Fz, F4, FC3, FCz, FC4, C3, Cz, and C4 were selected for analysis, because MMN responses are typically most prominent on fronto-central sites when referenced to the average of mastoids. To decrease the likelihood of bogus results, we created a grand-average waveform over all deviant minus standard responses of the nine pre-selected electrode sites to determine the peak latency of the MMN response (Luck \& Gaspelin, 2017). A \pm 20 millisecond time window centred at this average latency of $334 \mathrm{~ms}$ was then used to quantify the mean MMN amplitude from individual deviant minus standard difference waveforms.

\subsubsection{Source localisation}

To localise the sources of the MMN responses, the EEG data were pre-processed in an appropriate way for source imaging (Michel \& Brunet, 2019) with BESA Research 7.0 software (BESA GmbH, Gräfelfing, Germany). The data were filtered with a band pass of $0.1-40 \mathrm{~Hz}$ (slope $24 \mathrm{~dB} /$ octave) and then divided into epochs using a -100 to $800 \mathrm{~ms}$ time window relative to the onset of the stimuli. Epochs exceeding $\pm 100 \mu \mathrm{V}$ during a $200 \mathrm{~ms}$ time window centred at the MMN latency (234-434 ms) were rejected. The remaining epochs were averaged separately for each stimulus type. Average reference was used for making deviantminus standard waveforms. Source localisation was conducted only for the participants who had a minimum of 80 epochs remaining for game stimuli, non-game stimuli, or both. For each remaining participant, the latencies of the most negative peaks in 284-384 ms time window were determined. A head model for 10-year-olds from Besa Research 7.0 was used for source modelling. Two dipoles were fitted over left and right auditory cortices in $40 \mathrm{~ms}$ time windows centred at these individual peaks of the game and the non-game condition. To be included in further analysis, the goodness of fit of the two-dipole model had to be at least 
$75 \%$ and at least one of the sources had to be in the auditory cortex or its proximity. For the game condition, 17 participants, and for the non-game condition, seven participants fulfilled these criteria. Since few participants showed an MMN response for the non-game condition, its sources are not further examined. For the game condition, 14 participants showed sources in the left auditory cortex and 15 participants in the right auditory cortex. The source strengths were measured as absolute values in $40 \mathrm{~ms}$ time windows centred at the strongest individual peaks found in the MMN time range of 284-384 ms.

\subsection{Training}

Between the last two EEG recording sessions, the children practiced some frequent English words (e.g. child, drink, high) with the "Say it again, kid" (SIAK) language learning game (Karhila et al., 2017; Ylinen \& Kurimo, 2017; see also Ylinen et al., submitted, for a newer mobile version of the game) on either Windows laptops or Android tablets using a headset. SIAK is a computer-based game for learning spoken English language. Playing the game is based on listening to spoken English words and imitating them as accurately as possible. SIAK uses automatic speech recognition (ASR) technology optimised for children's voices to evaluate children's utterances (Karhila et al., 2019, 2017). The game is designed as a computer board game and it has 27 levels. Each level is presented as a game board which the players can explore by moving their avatar around it. Game boards contain cards, which pop up when the players move their avatar on them. Each card has a picture, and when it pops up, the children hear the corresponding word first in Finnish and after that in English. When a microphone symbol is shown, the children are supposed to imitate the English word as accurately as they can. Players' utterances are recorded and sent to a game server where the ASR assesses each speech sound of the utterance. Then the players hear their own utterances played back to them, which is followed by the original English speaker's utterance for comparison. Finally, one to five stars are awarded to the player based on the ASR assessment. In the end of each level, the players have the chance to test their learning with a test card where a word learned at the level is produced without a model. New levels of the game are unlocked when at least three stars are scored from the test card word. If the players do not score enough stars, they can go back to the learning card to practise the word and then try the test card again. 
The children played all 27 game levels during a gaming period that lasted on average 4.3 weeks. They played the game on average 15.5 minutes a day 2.9 days a week. There were, however, only 6 levels that included words with the target phonemes ( 3 levels of /ð/ practise and 3 levels of $/ \theta /$ practise) of this study. That is, the target phonemes were not presented elsewhere in the game, although the game was played considerably longer than the target words and phonemes were rehearsed. To investigate the effects of game-based learning, we compared learning between two different learning conditions: a game condition and a nongame condition. The game condition had three normal SIAK game levels as described above, with words including either of the target sounds. By comparison, the non-game condition had three non-game levels with words including the other target sound. These levels were embedded within the game after the above-mentioned three game levels. However, the nongame levels were stripped of all the game-like elements, that is, the colourful game board, freedom to explore, and gaining stars. Instead, the non-game condition had a white screen with a black arrow and had a forced presentation order of the target words (Figure 2). Similarly to the game condition, the task was to imitate the heard English words. Thus, the only difference between the game and non-game training was the presence or lack of gaming elements, which included the visual presentation of the game, freedom to explore the game and make choices, and rewards (i.e., stars provided by ASR for feedback).

To eliminate any effect of phoneme difficulty on the comparison between game and nongame conditions, the use of words with voiced or voiceless fricatives was counterbalanced between conditions and participants. Specifically, 18 of the participants practiced the word containing the phoneme /ð/ with the game levels and the phoneme $/ \theta /$ with the non-game levels, whereas the other 19 participants practiced the word containing the phoneme $/ \theta$ / with the game levels and the phoneme/ð/ with the non-game levels. To match the exposure to the target words and speech sounds in the game and non-game levels, the number of times the cards containing words with the target phonemes were opened in the game levels was recorded and the matching number of target words was presented in the non-game levels (to allow free exploration of the game levels, they had to be presented prior to the non-game levels, as we did not wish the non-game to restrict playing; therefore the order of the game and non-game levels could not, unfortunately, be counterbalanced). Except for the non-game, the players were free to explore the game condition levels as they wished. 

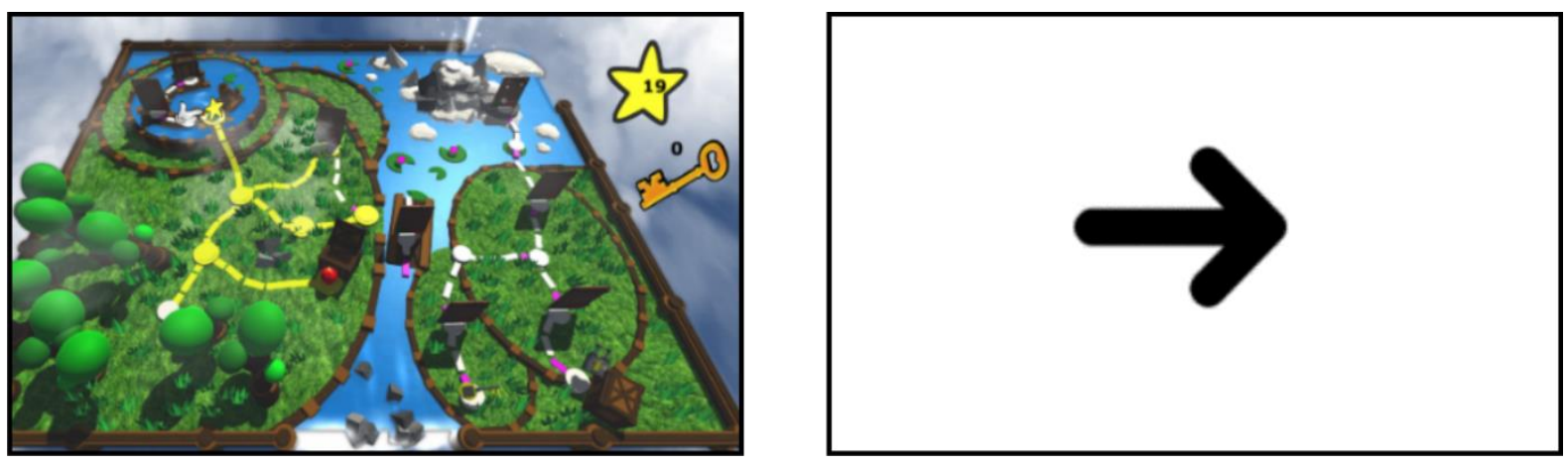

Figure 2. Screen shots of game and non-game levels.

\subsection{Statistical analyses}

The game and non-game differences over the measurement time points were compared with linear mixed model analyses with IBM SPSS Statistics, Version 25. Two linear mixed model analyses were conducted separately. As the children were likely to hear English, and more specifically, the target phonemes of this study from other sources in addition to the game between the measurements, we compared the change in MMN amplitude between the baseline measurement and the pre-measurement. The effects of out-of-game exposure to English language and hearing the stimulus sequences during the first measurement were analysed for the 22 participants who participated in the baseline MMN measurement. The analysis included Treatment (game - non-game), Anteriority, and Laterality as factors and Time between the baseline measurement and pre-measurement in days as a covariate.

Participant and treatment were included as random effects, and scaled identity was chosen as the covariance structure based on Schwarz's Bayesian Criterion (BIC). To compare the effectiveness of the game and non-game training, a mixed model analysis for all 37 participants' MMN measures pre and post training was conducted. The analysis included the same factors as the previously described one (Treatment, Anteriority, and Laterality), and the Time between the pre and post measurements in days was included as a covariate. Participant and Treatment were included as random effects and unstructured covariance matrix was chosen based on BIC. Only the significant main effects and interactions at alpha level 0.05 including Treatment or Time are reported in the results.

The strength of left and right temporal sources of the MMN response was compared with linear mixed model analysis, where Hemisphere was included as a factor. 


\section{Results}

Before participating in the first EEG recording session, none of the children knew the meanings of the stimulus words. After training with the game, 19 children had learned the meanings of the stimulus words correctly. Training with the non-game resulted in 16 children learning the stimulus word meanings correctly.

None of the main effects or interactions were significant in the linear mixed model analysis for the effects of out-of-game exposure to English language and hearing the same stimuli from the first measurement repeatedly.

The linear mixed model analysis for game vs. non-game effects revealed a significant interaction of Treatment and Time, $\mathrm{F}(1,1195.08)=26.81, p=<0.001$. To adjust for the different number of days between the pre-measurement and the post-measurement between participants, the estimated marginal means for the game and non-game were evaluated at the day of the pre-measurement and at the mean of days between pre and post measurements (36.62 days), as presented in Table 1. Training with the game condition increased the MMN amplitude more than training with the non-game condition (Figures $3 \& 4$, Table 1). Further pairwise comparison revealed a post-measurement difference between the game and the nongame condition's estimated marginal means ( $p=0.008,95 \% \mathrm{CI}=0.19,1.20)$. The $\mathrm{MMN}$ amplitude was more negative for the game condition $(-0.99 \mu \mathrm{V})$ than for the non-game condition $(-0.30 \mu \mathrm{V})$.

\begin{tabular}{lllccc}
\hline & & & & \multicolumn{2}{c}{$95 \%$ CI } \\
\cline { 5 - 6 } Condition & Time point & EMM & S.E. & Lower & Upper \\
\hline Game & pre-measurement & -0.32 & 0.22 & -0.76 & 0.13 \\
& post-measurement & -0.99 & 0.22 & -1.44 & -0.54 \\
\multirow{2}{*}{ Non-game } & pre-measurement & -0.25 & 0.22 & -0.70 & 0.20 \\
& post-measurement & -0.30 & 0.22 & -0.74 & 0.15 \\
\hline
\end{tabular}

Table 1. Estimated marginal means (EMM) of the MMN amplitudes in $\mu \mathrm{V}$ for the game and non-game conditions in the pre and post measurements. Time point pre-measurement represents the estimated mean at the day of the pre-measurement and the post-measurement represents the estimated mean at 36.62 days, which is the mean of days between the premeasurement and the post-measurement. 
Game

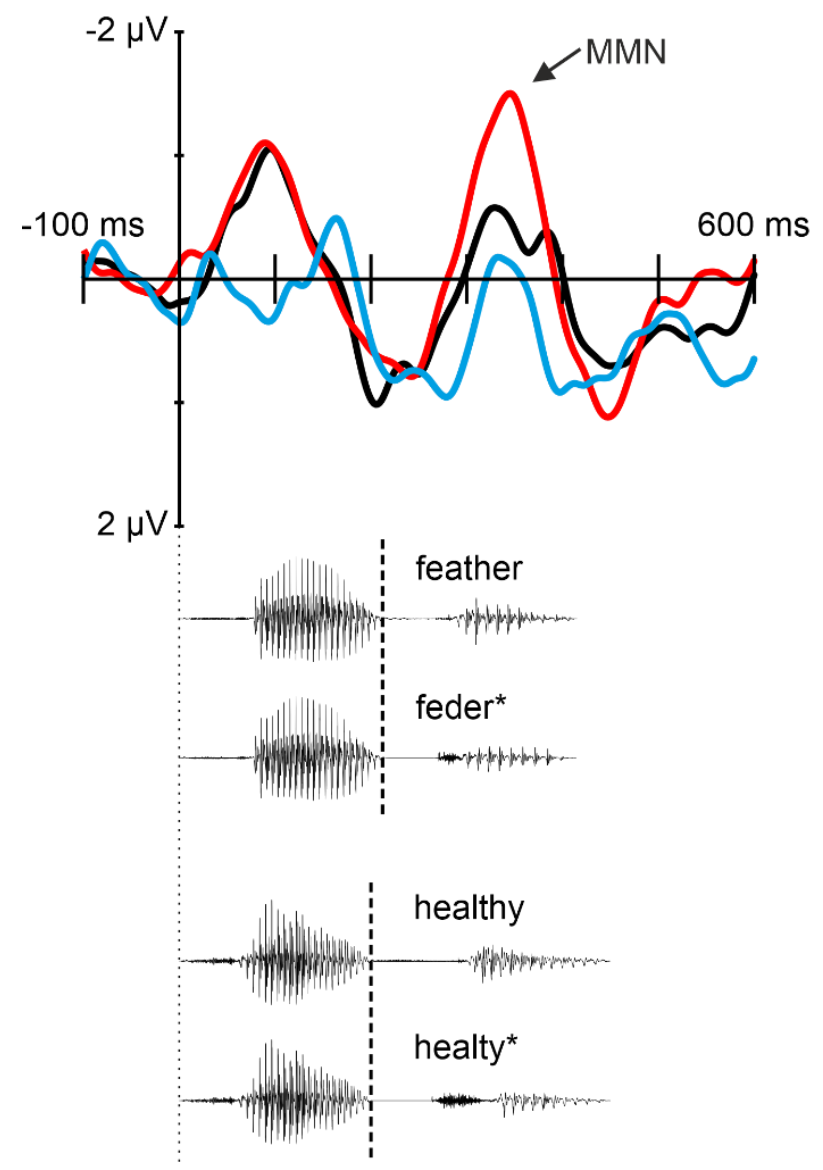

Non-game

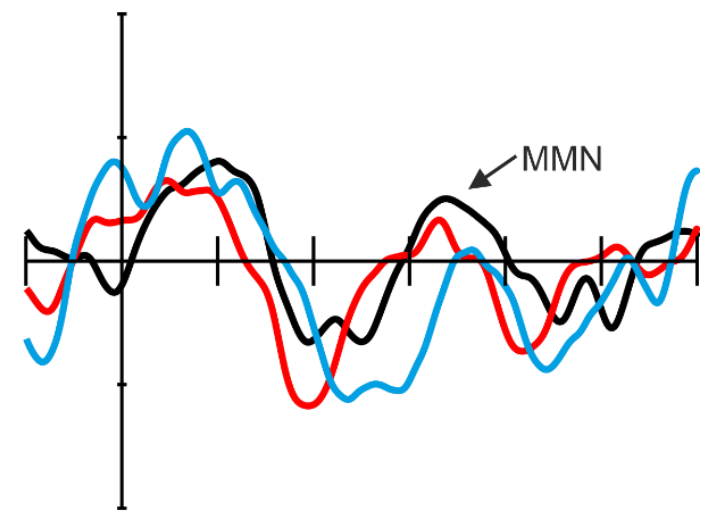

Baseline measurement

Pre-training

Post-training

Figure 3. The MMN responses at electrode FCz at baseline measurement, pre-training, and post-training, and the waveforms of the stimulus sounds presented on the same time scale. Deviation points between standard and deviant stimuli are marked with dashed lines. The average of both stimulus word-pseudoword pairs were used in the non-game and game conditions. 


\section{Game}

Baseline

\section{Pre-training}

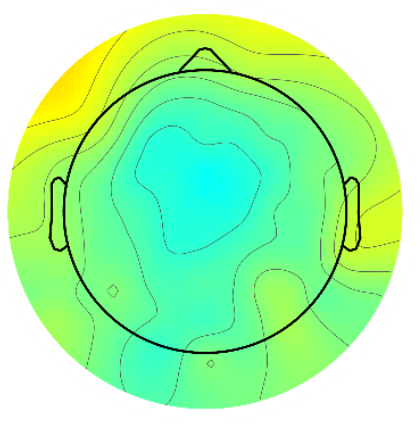

\section{Post-training}

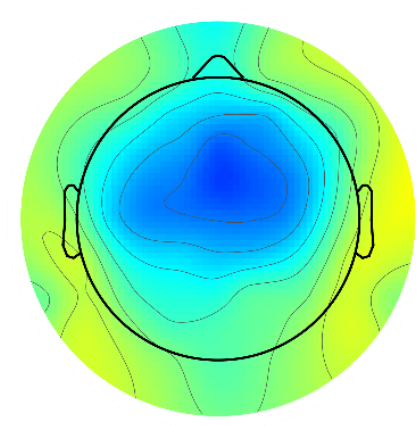

Non-game
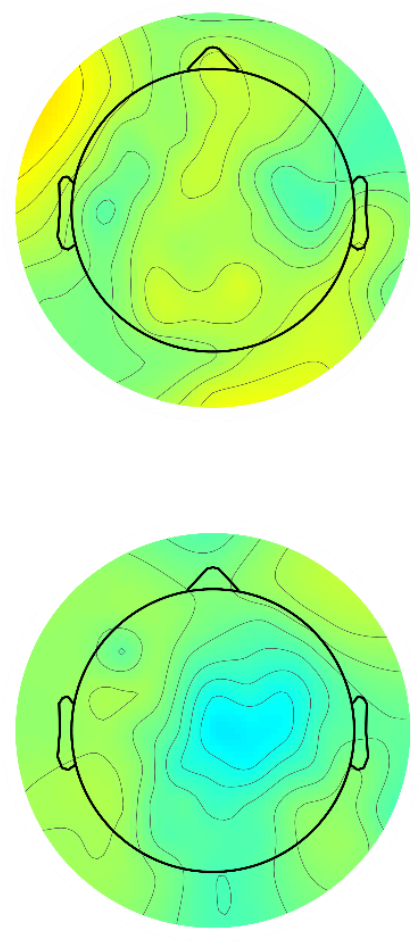

1

0.5

0

$-0.5$

$-1$

$-1.5$

$-2$

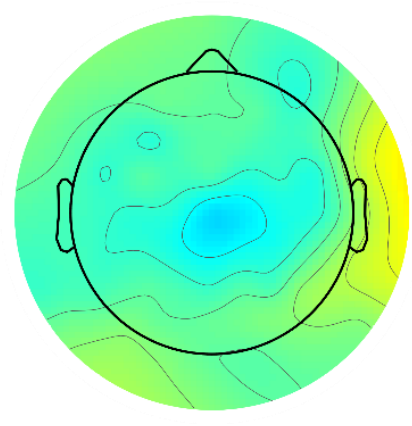

Figure 4. Scalp topography maps for the MMN responses at baseline measurement, pretraining, and post-training.

The linear mixed model analysis revealed no significant difference between the strengths of the left $(\mathrm{M}=25.20, \mathrm{SD}=10.38,95 \% \mathrm{CI}=16.94,33.45)$ and right $(\mathrm{M}=31.90, \mathrm{SD}=18.36$, $95 \% \mathrm{CI}=23.93,39.88)$ temporal sources of the game condition MMN response. The left temporal source was found in 14 participants and the right temporal source in 15 participants. An example of the locations of the dipoles of one participant is presented in Figure 5. 

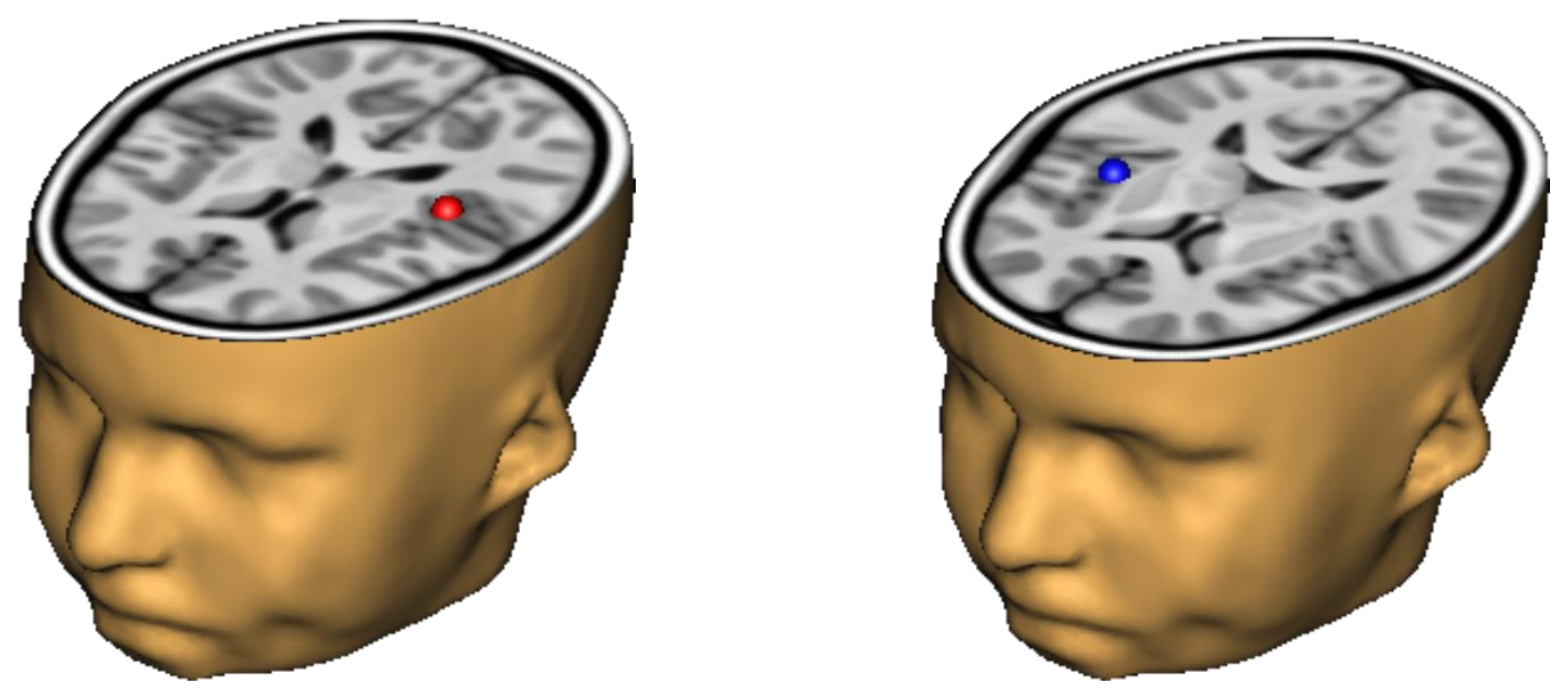

Figure 5. Left and right temporal sources of a representative participant for post-training game condition depicted on an age-appropriate MRI template.

\section{Discussion}

The present study aimed to investigate the effectiveness of digital-game based learning approach on children's foreign language learning by using the MMN component of ERP as an indicator of training-induced plastic changes in the brain function (see Ylinen et al., 2010). More specifically, we compared the effects of game-based learning to the effects of nongame-based approach with equal number or repetitions. The MMN responses increased more for the words learned with the game than for the words learned with the non-game.

It is well-established that the MMN is sensitive to acoustic differences. The finding of larger MMN for words learned with the game than with the non-game cannot, however, be explained by differences in saliency of the acoustic stimulus properties, because our main finding is based on an interaction between Time and Treatment, and no differences were observed before training to the same words. The effect cannot be due to phoneme or word difficulty either, since the words that were learned with the game or with the non-game were counterbalanced between participants and the MMN responses were formed across both words in each condition. Therefore, there was, on average, no difference between the stimuli used for the game and the non-game MMN responses. Thus, stimulus properties cannot account for the pattern of results. 
Many previous studies that have investigated the effectiveness of digital game-based learning have compared two participant groups: one learning with a game and another learning with some other activity or another type of game (Aghlara \& Tamjid, 2011; deHaan et al., 2010; Franciosi, 2017; Hsu, 2017; Liu \& Chu, 2010; Yip \& Kwan, 2006; Young \& Wang, 2014). Although this is general practise, it is difficult to fully exclude the possibility that individual differences between the participants' learning abilities result in different learning effects between groups despite similar pre-test performance. This considers especially studies in children's learning, because of the large differences in the maturation of children's skills. In the present study, however, the effect of individual learning-ability differences was controlled for: All participants learned a target word with the game and another word with the nongame. Hence, the different results for the game and the non-game do not stem from differences in learning abilities.

Taken together, since the properties of the stimuli or the children's learning abilities do not account for the larger post-test than pre-test MMN for the game condition, the most plausible explanation for the pattern of results is that the game is more effective for learning than the non-game. The results of this study about beneficial effects of gaming on foreign-language learning are in line with previous studies (Aghlara \& Tamjid, 2011; Franciosi, 2017; Hsu, 2017; Liu \& Chu, 2010; Sandberg et al., 2014; Tsai \& Tsai, 2018; Young \& Wang, 2014) However, while some previous studies (Franciosi, 2017; Young \& Wang, 2014) did not control for practising time that is likely to enhance learning (e.g. Tejedor-García et al., 2020), it is not fully clear whether their gaming benefits are due to the increase of practising time or some gaming elements. To this end, the present study sought to investigate the effect of gaming compared to similar amount of non-game practise to determine the effect of gaming elements. In the present study, the number of times each target word was trained was equal between the game and the non-game conditions. Further, the number of times other words containing the target phonemes were trained was also equal between the conditions. The enhancement in the MMN amplitude for game-trained words cannot, therefore, be explained by different playing times of game and non-game conditions. Rather, the effect must be due to the gaming elements per se benefiting learning.

The conclusion that gaming elements benefit learning raises a further question, specifically which elements are the most important ones. The present study investigated the effects of game-based learning by comparing it to learning with a non-game condition that had been 
stripped of all game-like elements, that is, game graphics, freedom to explore, and feedback rewards. That is, any of these could drive learning or their effects might accumulate. For example, feedback is one of the elements that have been suggested to be relevant for player enjoyment in games (Sweetser \& Wyeth, 2005). Previous studies have also shown that feedback contributes positively to learning results (e.g. Erhel \& Jamet, 2013). Gaming has also been found to improve attention (Oei \& Patterson, 2013), which in turn might affect learning positively. Unfortunately, the current study design did not allow us to tease apart the effects of feedback and the other gaming elements. Therefore, in the future it would be interesting to further explore the effectiveness of different game elements separately to determine their contribution to the learning effects of gaming.

As compared with the previous studies, the present study also expands the knowledge of the learning effects of gaming by demonstrating gaming-induced plastic changes in the brain function. Whereas previous studies have shown that gaming has benefits on pronunciation (Young \& Wang, 2014), vocabulary (Aghlara \& Tamjid, 2011; Hsu, 2017; Sandberg et al., 2014), and listening and speaking skills (Liu \& Chu, 2010), this study demonstrates how gaming can benefit learning by inducing changes in the auditory cortex, which are most likely due to the establishment of long-term memory traces for foreign speech-sounds and words (see Ylinen et al., 2010). The finding that roughly half of the children learned the word meanings during gaming lends some support on this interpretation, although MMN measurement and vocabulary test inevitably measure different aspects of language learning. The MMN reflects, among other processes, the pre-attentive activation of long-term memory traces for familiar words (Pulvermüller et al., 2001; Shtyrov et al., 2008) and speech sounds (Näätänen et al., 1997; Ylinen et al., 2010). Therefore, a larger MMN response after than before gaming likely indicates that the auditory word forms of those words or speech sounds were learned and the post-test difference between conditions suggests that gaming induced stronger long-term memory traces than training with the non-game. However, it is noteworthy that the meanings of the words were learned almost equally often with the nongame training (16 vs. 19 for non-game vs. game, respectively), despite of MMN responses being weaker for the non-game. Perhaps, then, gaming resulted in higher degree of automaticity of processing during the short training period, whereas non-game training resulted in learning that is more dependent on attentional resources. 
Although we expected to find stronger MMN source in the right hemisphere (Nora et al., 2017; S. Ylinen et al., 2019), source localisation revealed no significant differences between the strength of the left and right temporal sources of the MMN response in the game condition. Even though the right hemisphere has been shown to be related to foreign word processing in children (Nora et al., 2017; S. Ylinen et al., 2019), language processing in children has also been demonstrated to shift to the left hemisphere as age increases (McNealy et al., 2011), individual differences in this shift process could explain why no hemispheric difference was found. It was not possible to compare the sources for the game responses and the non-game responses, because too few participants showed an MMN response for the words learned with the non-game. The absence of significant MMN responses in this condition suggests, firstly, that our short non-game training was not as effective as the game training in establishing long-term memory representations for foreign speech-sounds or words, and, secondly, that subtle acoustic differences between the stimuli were not acoustically salient enough to elicit MMNs consistently.

To conclude, the results of this study show that gaming may have a positive effect on learning. Gaming induced more robust plastic changes in brain function, than training with the non-game. The long-term memory traces for words learned with the game were stronger, as revealed by the increased MMN amplitude. Thus, gaming can benefit children's learning, and, it is the gaming elements themselves that support learning.

\section{Conflict of Interest}

After the closure of the research project where the experiments were conducted, Anna-Riikka Smolander and Reima Karhila founded a company called Hokema Oy, that attempts to commercialize the game and speech technology developed in this and the preceding research projects. In addition to Smolander and Karhila, Sari Ylinen and Mikko Kurimo have been listed as inventors. This has had no effect on the results of the current study or their interpretation.

\section{Author contributions}

Katja Junttila: Conceptualization, Methodology, Formal analysis, Investigation, Data Curation, Writing - Original Draft, Visualization, Funding acquisition; Anna-Riikka 
Smolander: Software, Investigation, Data Curation; Reima Karhila: Methodology, Software; Anastasia Giannakopoulou: Investigation; Maria Uther: Investigation; Mikko

Kurimo: Methodology, Funding acquisition; Sari Ylinen: Conceptualization, Methodology, Writing - Review \& Editing, Supervision, Project administration, Funding acquisition

\section{Acknowledgements}

We wish to thank our participants, their caregivers, and their schools, principals, and teachers for collaboration; City of Helsinki Education Division, City of Espoo Education and Cultural Services, and City of Vantaa Education Department for access to schools; Vertti Viitanen, Aleksander Nikulin, Olli Rantula, and Krupakar Dhinakaran for programming the game; Seppo Enarvi and Kalle Palomäki for participating in developing the ASR technology; Nella Moisseinen, Mari Falcon, and Pietari Nurmi for their assistance in data collection; Cintia Widmann Etchemaite, Kari Lehikoinen, Anniina Åkerla, and Marika Pajunen for their assistance in data processing; Tommi Makkonen for technical assistance, and Jari Lipsanen for statistical assistance.

\section{Funding}

This work was supported by the Academy of Finland (projects no 274058 and 274075), the Doctoral Programme in Psychology, Learning and Communication, and the University of Helsinki Research Funds.

\section{References}

Acquah, E. O., \& Katz, H. T. (2020). Digital game-based L2 learning outcomes for primary through high-school students: A systematic literature review. Computers and Education, 143. https://doi.org/10.1016/j.compedu.2019.103667

Aghlara, L., \& Tamjid, N. H. (2011). The effect of digital games on Iranian children's vocabulary retention in foreign language acquisition. Procedia - Social and Behavioral Sciences, 29, 552-560. https://doi.org/10.1016/j.sbspro.2011.11.275

Baker, J. M., Martin, T., Aghababyan, A., Armaghanyan, A., \& Gillam, R. (2015). Cortical Activations During a Computer-Based Fraction Learning Game: Preliminary Results 
from a Pilot Study. Technology, Knowledge and Learning, 20(3), 339-355.

https://doi.org/10.1007/s10758-015-9251-y

Bavelier, D., \& Davidson, R. J. (2013). Games to do you good. Nature, 494, 425-426. https://doi.org/https://doi.org/10.1038/494425a

Boersma, P., \& Weenink, D. (2010). Praat: Doing phonetics by computer [Computer program]. (Version 5.1.45). http://www.praat.org/

Chen, M. H., Tseng, W. T., \& Hsiao, T. Y. (2018). The effectiveness of digital game-based vocabulary learning: A framework-based view of meta-analysis. British Journal of Educational Technology, 49(1), 69-77. https://doi.org/10.1111/bjet.12526

Cheour, M., H.T. Leppänen, P., \& Kraus, N. (2000). Mismatch negativity (MMN) as a tool for investigating auditory discrimination and sensory memory in infants and children. Clinical Neurophysiology, 111(1), 4-16. https://doi.org/10.1016/S1388-2457(99)001911

deHaan, J., Michael Reed, W. M., \& Kuwada, K. (2010). The effect of interactivity with a music video game on second language vocabulary recall. Language Learning and Technology, 14(2), 74-94. http://llt.msu.edu/vol14num2/dehaanreedkuwada.pdf

Eichenbaum, A., Bavelier, D., \& Green, S. (2014). Video Games: Play That Can Do Serious Good. American Journal of Play, 7(1), 50-72.

Erhel, S., \& Jamet, E. (2013). Digital game-based learning: Impact of instructions and feedback on motivation and learning effectiveness. Computers and Education, 67, 156167. https://doi.org/10.1016/j.compedu.2013.02.019

Fitzgerald, K., \& Todd, J. (2020). Making Sense of Mismatch Negativity. In Frontiers in Psychiatry (Vol. 11). Frontiers Media S.A. https://doi.org/10.3389/fpsyt.2020.00468

Franciosi, S. J. (2017). The Effect of Computer Game-Based Learning on FL Vocabulary Transferability. Source: Journal of Educational Technology \& Society, 20(1), 123-133. 
https://doi.org/10.2307/jeductechsoci.20.1.123

Grgurović, M., Chapelle, C. A., \& Shelley, M. C. (2013). A meta-analysis of effectiveness studies on computer technology-supported language learning. ReCALL, 25(2), 165-198. https://doi.org/10.1017/S0958344013000013

Hong, J. S., Han, D. H., Kim, Y. I., Bae, S. J., Kim, S. M., \& Renshaw, P. (2017). English language education on-line game and brain connectivity. ReCALL, 29(1), 3-21. https://doi.org/10.1017/S0958344016000173

Hsu, T. C. (2017). Learning English with Augmented Reality: Do learning styles matter? Computers and Education, 106, 137-149. https://doi.org/10.1016/j.compedu.2016.12.007

Kalyakin, I., González, N., Joutsensalo, J., Huttunen, T., Kaartinen, J., \& Lyytinen, H. (2007). Optimal Digital Filtering versus Difference Waves on the Mismatch Negativity in an Uninterrupted Sound Paradigm. Developmental Neuropsychology, 31(3), 429-452. https://doi.org/10.1080/87565640701229607

Karhila, R., Smolander, A. R., Ylinen, S., \& Kurimo, M. (2019). Transparent pronunciation scoring using articulatorily weighted phoneme edit distance. Proceedings of the Annual Conference of the International Speech Communication Association, INTERSPEECH, 2019-Septe, 1866-1870. https://doi.org/10.21437/Interspeech.2019-1785

Karhila, R., Ylinen, S., Enarvi, S., Palomäki, K., Nikulin, A., Rantula, O., Viitanen, V., Dhinakaran, K., Smolander, A. R., Kallio, H., Junttila, K., Uther, M., Hämäläinen, P., \& Kurimo, M. (2017). SIAK - A game for foreign language pronunciation learning. Proceedings of the Annual Conference of the International Speech Communication Association, INTERSPEECH, 2017-Augus, 3429-3430. https://doi.org/10.21437/Interspeech.2017-2046

Liu, T. Y., \& Chu, Y. L. (2010). Using ubiquitous games in an English listening and speaking course: Impact on learning outcomes and motivation. Computers and Education, 55(2), 630-643. https://doi.org/10.1016/j.compedu.2010.02.023 
Luck, S. J., \& Gaspelin, N. (2017). How to get statistically significant effects in any ERP experiment (and why you shouldn't). Psychophysiology, 54(1), 146-157. https://doi.org/10.1111/psyp.12639

Mayer, R. E. (2015). On the Need for Research Evidence to Guide the Design of Computer Games for Learning. In Educational Psychologist (Vol. 50, Issue 4, pp. 349-353). Routledge. https://doi.org/10.1080/00461520.2015.1133307

McNealy, K., Mazziotta, J. C., \& Dapretto, M. (2011). Age and experience shape developmental changes in the neural basis of language-related learning. Developmental Science, 14(6), 1261-1282. https://doi.org/10.1111/j.1467-7687.2011.01075.x

Michel, C. M., \& Brunet, D. (2019). EEG source imaging: A practical review of the analysis steps. Frontiers in Neurology, 10(APR). https://doi.org/10.3389/fneur.2019.00325

Näätänen, R., Gaillard, A. W. K., \& Mäntysalo, S. (1978). Early selective-attention effect on evoked potential reinterpreted. Acta Psychologica, 42(4), 313-329. https://doi.org/10.1016/0001-6918(78)90006-9

Näätänen, R., Paavilainen, P., Rinne, T., \& Alho, K. (2007). The mismatch negativity $(\mathrm{MMN})$ in basic research of central auditory processing: A review. Clinical Neurophysiology, 118(12), 2544-2590. https://doi.org/10.1016/j.clinph.2007.04.026

Näätänen, Risto, Lehtokoski, A., Lennes, M., Cheour, M., Huotilainen, M., Iivonen, A., Vainio, M., Alku, P., Ilmoniemi, R. J., Luuk, A., Allik, J., Sinkkonen, J., \& Alho, K. (1997). Language-specific phoneme representations revealed by electric and magnetic brain responses. Nature, 385(6615), 432-434. https://doi.org/10.1038/385432a0

Nenonen, S., Shestakova, A., Huotilainen, M., \& Näätänen, R. (2005). Speech-sound duration processing in a second language is specific to phonetic categories. Brain and Language, 92(1), 26-32. https://doi.org/10.1016/j.bandl.2004.05.005

Nora, A., Karvonen, L., Renvall, H., Parviainen, T., Kim, J. Y., Service, E., \& Salmelin, R. (2017). Children show right-lateralized effects of spoken word-form learning. PLoS 
ONE, 12(2), 1-20. https://doi.org/10.1371/journal.pone.0171034

Oei, A. C., \& Patterson, M. D. (2013). Enhancing Cognition with Video Games: A Multiple Game Training Study. PLoS ONE, 8(3). https://doi.org/10.1371/journal.pone.0058546

Peterson, M. (2010a). Computerized games and simulations in computer-assisted language learning: A meta-analysis of research. Simulation and Gaming, 41(1), 72-93. https://doi.org/10.1177/1046878109355684

Peterson, M. (2010b). Massively multiplayer online role-playing games as arenas for second language learning. Computer Assisted Language Learning, 23(5), 429-439. https://doi.org/10.1080/09588221.2010.520673

Picton, T. W., Alain, C., Otten, L., Ritter, W., \& Achim, A. (2000). Mismatch negativity: Different water in the same river. In Audiology and Neuro-Otology (Vol. 5, Issues 3-4, pp. 111-139). S. Karger AG. https://doi.org/10.1159/000013875

Pulvermüller, F., Kujala, T., Shtyrov, Y., Simola, J., Tiitinen, H., Alku, P., Alho, K., Martinkauppi, S., Ilmoniemi, R. J., \& Näätänen, R. (2001). Memory traces for words as revealed by the mismatch negativity. NeuroImage, 14(3), 607-616. https://doi.org/10.1006/nimg.2001.0864

Sandberg, J., Maris, M., \& De Geus, K. (2011). Mobile English learning: An evidence-based study with fifth graders. Computers and Education, 57(1), 1334-1347. https://doi.org/10.1016/j.compedu.2011.01.015

Sandberg, J., Maris, M., \& Hoogendoorn, P. (2014). The added value of a gaming context and intelligent adaptation for a mobile learning application for vocabulary learning. Computers and Education, 76, 119-130. https://doi.org/10.1016/j.compedu.2014.03.006

Shtyrov, Y., Osswald, K., \& Pulvermüller, F. (2008). Memory traces for spoken words in the brain as revealed by the hemodynamic correlate of the mismatch negativity. Cerebral Cortex, 18(1), 29-37. https://doi.org/10.1093/cercor/bhm028 
Sinkkonen, J., \& Tervaniemi, M. (2000). Towards Optimal Recording and Analysis of the Mismatch Negativity. In Audiol Neurootol (Vol. 5). www.karger.com

Sweetser, P., \& Wyeth, P. (2005). GameFlow: A Model for Evaluating Player Enjoyment in Games. In ACM Computers in Entertainment (Vol. 3, Issue 3).

Tejedor-García, C., Escudero-Mancebo, D., Cardeñoso-Payo, V., \& González-Ferreras, C. (2020). Using Challenges to Enhance a Learning Game for Pronunciation Training of English as a Second Language. IEEE Access, 8, 74250-74266. https://doi.org/10.1109/ACCESS.2020.2988406

Tsai, Y. L., \& Tsai, C. C. (2018). Digital game-based second-language vocabulary learning and conditions of research designs: A meta-analysis study. Computers and Education, 125, 345-357. https://doi.org/10.1016/j.compedu.2018.06.020

Wechsler, D. (2010). WISC-IV - Wechsler Intelligence Scale for Children - IV. Psykologien Kustannus Oy.

Yip, F. W. M., \& Kwan, A. C. M. (2006). Online vocabulary games as a tool for teaching and learning English vocabulary. Educational Media International, 43(3), 233-249. https://doi.org/10.1080/09523980600641445

Ylinen, S., Junttila, K., Laasonen, M., Iverson, P., Ahonen, L., \& Kujala, T. (2019). Diminished brain responses to second-language words are linked with native-language literacy skills in dyslexia. Neuropsychologia, 122. https://doi.org/10.1016/j.neuropsychologia.2018.11.005

Ylinen, Sari, \& Kurimo, M. (2017). Kielenoppiminen vauhtiin puheteknologian avulla. In H. Savolainen, R. Vilkko, \& L. Vähäkylä (Eds.), Oppimisen tulevaisuus (pp. 57-69). Gaudeamus.

Ylinen, Sari, Smolander, A.-R., Karhila, R., Kakouros, S., \& Kurimo, M. (n.d.). The effects of a digital articulatory game on the ability to perceive 1 speech-sound contrasts in another language. www.pop2talk.com 
Ylinen, Sari, Uther, M., Latvala, A., Vepsäläinen, S., Iverson, P., Akahane-Yamada, R., \& Näätänen, R. (2010). Training the Brain to Weight Speech Cues Differently: A Study of Finnish Second-language Users of English. Journal of Cognitive Neuroscience, 22(6), 1319-1332. https://doi.org/10.1162/jocn.2009.21272

Young, S.-S.-C., \& Wang, Y.-H. (2014). The Game Embedded CALL System to Facilitate English Vocabulary Acquisition and Pronunciation. In Educational Technology \& Society (Vol. 17, Issue 3).

Zou, D., Huang, Y., \& Xie, H. (2019). Digital game-based vocabulary learning: where are we and where are we going? Computer Assisted Language Learning, 1-27. https://doi.org/10.1080/09588221.2019.1640745 